\title{
Dosaggio alluminio plasmatico: proposta di modello organizzativo
}

\author{
L. Scarpino', A. Confessore', A. Bruno ${ }^{2}$, C. D'Alessandro² \\ ${ }^{1}$ Servizio Emodialisi USL N. 2 Castrovillari (CS) \\ ${ }^{2}$ Laboratorio di Chimica USL N. 2 Castrovillari (CS)
}

l sovraccarico e la tossicità dell'alluminio rappresentano un grave rischio per i pazienti con Insufficienza Renale Cronica, soprattutto per i pazienti in dialisi.

I fattori responsabili di tale predisposizione sono:

- mancanza di eliminazione renale;

- aumentato assorbimento conseguente alle alterazioni della parete gastroenterica dovute all'uremia $(1,2)$;

- utilizzo di chelanti per il fosforo a base di $\left.\mathrm{Al}(\mathrm{OH})_{3}\right)$

- presenza dell'alluminio nel liquido di dialisi (anche dialisi peritoneale ed emofiltrazione) ed in molti farmaci e soluzioni terapeutiche.

Oltre alla possibile esposizione legata a motivi "iatrogeni" esistono altre fonti di intossicazione quali il contenuto di alluminio nell'acqua potabile, l'uso di pentole e fogli per uso domestico in alluminio, consumo di bibite in lattina.

Dal punto di vista clinico posiamo avere forme con interessamento del SNC (encefalopatia con disprassia, convulsioni, demenza), anemia microcitica ed osteopatia grave; molti organi possono comunque essere interessati. Le forme più gravi, generalmente conseguenti ad un'esposizione attraverso il liquido di dialisi, oggi sono pressoché scomparse. La conoscenza del problema "alluminio", la deionizzazione efficace dell'ac- qua destinata alla preparazione, il minore utilizzo di chelanti con $\mathrm{Al}(\mathrm{OH})_{3}$ e la possibilità di eseguire i controlli sul siero e sui liquidi di interesse terapeutico hanno portato alla riduzione notevole della patologia da alluminio. Ciò nonostante non possiamo considerare tale patologia un problema risolto, in quanto vi sono ancora pazienti che possono presentare problemi clinici (soprattutto ossei) legati al sovraccarico dell'elemento, pur senza presentare la grave sindrome descritta in passato.

Il dosaggio dell'alluminio nel plasma dei pazienti e/o nei liquidi di interesse terapeutico pone dei seri problemi sia in sede di campionamento (prelievi) che in sede di analisi chimica (dosaggio).

Il campionamento è potenzialmente la maggior sorgente di contaminazione per la presenza nell'ambiente di alluminio e pertanto occorre molta attenzione in questa fase.

Del resto, ogni passo del ciclo analitico deve essere valutato come una possibile fonte di contaminazione: provette, reattivi, vials dell'autocampionatore ecc.

Abbiamo eseguito test di rilascio sul materiale di consumo di diverse ditte, orientandoci su alcuni prodotti che hanno mostrato sufficiente garanzia, standardizzando le modalità di prelievo e di manipolazione del campione.

Relativamente al problema delle interfe- renze nella fase di lettura, considerazioni note ci dicono che i campioni biologici contengono una varietà di composti che possono interferire con la determinazione elettrotermica dell'Al-s.

Principalmente due composti sono particolarmente importanti ed hanno notevole rilevanza ai fini analitici: i cloruri ed il materiale organico (proteine e lipidi). I cloruri producono interferenza negativa, in quanto la formazione di cloruro di alluminio volatile provoca perdite dell'alluminio durante la fase di essiccamento ed incenerimento. Anche durante la fase di atomizzazione possono essere sottratti atomi allo stato elementare, con una complessiva riduzione dell'entità de! segnale. Proteine e lipidi possono produrre residui organici che interferiscono in modo causale nella misura dell'alluminio, come abbiamo riscontrato con alcuni campioni fortemente lipemici.

In letteratura viene proposta una varietà di modificatori chimici per ridurre i fenomeni di interferenza e migliorare il segnale. Tuttavia, ritenendo prioritaria la massima riduzione dei vari passaggi e manipolazioni, non abbiamo usato nessuno di questi prodotti, cercando di ottimizzare, in alternativa, un programma termico più drastico dato che l'alluminio permette più elevate temperature di atomizzazione. 
Lo scopo di questo lavoro è di affrontare il problema del dosaggio dell'alluminio nel plasma dei pazienti dializzati, proponendo il nostro modello organizzativo.

\section{Materiali e metodi}

\section{A) Pazienti:}

sono stati analizzati n. 406 campioni (203 coppie) relativi a $n$. 69 pazienti (43 $\mathrm{M} \mathrm{e} 26 \mathrm{~F}$ ) con età media 54.5 anni (range 21-81), età dialitica media di 48.1 mesi (range 3-135) in dialisi ospedaliera presso i centri di Castrovillari (CS) e Lungro (CS). In entrambi i centri l'acqua viene trattata con desalinizzatori (deionizzatori a scambio ionico) centralizzati; i monitor per la dialisi sono Monitral $1 \mathrm{e}$ Monitral S della ditta Hospal.

\section{B) Materiali:}

- provette in polistirene con tappo a tenuta (Falcon 2054);

- micropuntali 0-200 mcl (Oxford confezione singola);

- vials autocampionatore in politene (Varian);

\section{C) Apparecchiature:}

- spettrofotometro in Assorbimento Atomico con correttore al Deuterio Varian (mod. Spectra 30);

- fornetto di grafite Varian (mod. GTA96);

- autocampionatore Varian (mod. PSD-96);

- tubi pirolitici Varian;

- micropipetta Eppendorf $100 \mathrm{mcl}$;

- pipette $1 \mathrm{ml}$ Flow (confezione singola).

\section{D) Reattivi e Soluzioni:}

- acqua deionizzata (resistenza 20 megaohm);

- soluzione standard Al 1 mg/ml Merck;

- acido nitrico (tipo suprapur Merck);

- triton X-100 (Merck).

\section{E) Modalità di prelievo:}

i prelievi sono stati effettuati al mattino, a digiuno e dopo almeno tre giorni dalla sospensione dell'assunzione di prodotti a base di alluminio.

Poiché il prelievo può essere la maggiore fonte di contaminazione, occorre procedere con attenzione e cautela.

Come già riferito, abbiamo usato provette in polistirene con tappo a tenuta che si sono dimostrate adatte allo scopo. La procedura di prelievo è stata diversa nei pazienti che dovevano sottoporsi a dialisi nella seduta del mattino rispetto a quelli che, dovendo dializzare nel pomeriggio, venivano convocati al mattino in occasione dei prelievi mensili di controllo:

a) nel paziente che doveva sottoporsi a dialisi, dopo aver effettuato l'incannulamento, mediante ago-fistola, dell'accesso vascolare (Fistola Artero-Venosa) si lasciava defluire una quantità pari a 40-50 $\mathrm{ml}$ di sangue, attraverso l'agofistola, nella linea arteriosa del circuito, che connette tale ago al filtro di dialisi, nell'intento di pulire l'ago da eventuali fattori interferenti dovuti al materiale che lo compone (3); quindi si sconnetteva temporaneamente l'ago dalla linea arteriosa al fine di lasciare sgocciolare il sangue nelle due provette predisposte per il prelievo dell'Al-s; infine si riprendevano le normali procedure per permettere l'inizio del trattamento dialitico;

b) nel paziente che doveva sottoporsi ai controlli mensili (dialisi pomeridiana) veniva usato un ago tipo Butterfly (ago collegato ad un deflusso in materiale plastico) ed il prelievo per l'Al-s veniva effettuato per ultimo, dopo che erano passati nell'ago $25-30 \mathrm{ml}$ di sangue necessari per gli altri controlli periodici $(3,4)$.

In sintesi, dopo che nell'ago-fistola o ago Butterfly erano passati almeno 30 $\mathrm{ml}$ di sangue che lavano via gli eventuali fattori contaminanti, si lascia sgocciolare il sangue nelle provette in polistirene, avendo cura di non toccare i bordi delle provette.

Da ogni paziente venivano prelevate due provete con 4-5 $\mathrm{ml}$ di sangue ognuna.

I medici del reparto provvedevano a numerare, a caso, le provette in modo che l'operatore in laboratorio non fosse in grado di conoscere l'esatto accoppiamento (sapevano soltanto che di ogni paziente c'erano due provette). Le provette venivano inviate in serie giornaliere di 16-20 provette.

Prima di essere inviate al laboratorio, le provette venivano lasciate a riposare per almeno tre ore per favorire la "sieratu- ra" ed avere minori problemi in sede di analisi.

\section{Metodo di analisi}

Per valutare il grado di precisione del metodo sono stati organizzati due programmi di controllo di qualità interno:

a) i campioni dei pazienti, come già detto, erano in doppio e contrassegnati numericamente in modo che le coppie fossero cieche rispetto all'operatore;

b) la formazione in laboratorio di due pools di sieri a diversa concentrazione di alluminio; i due pool sono stati stoccati in provette, uguali a quelle utilizzate per i campioni, e congelati; una provetta al giorno di ogni livello veniva inserita nella serie giornaliera.

I campioni vengono analizzati entro 24 ore dal prelievo con uno standdard di calibrazione preparato al momento. Il campione viene diluito 1:4 con una soluzione di Triton X-100 allo $0.05 \%$. La stessa soluzione è utilizzata come bianco e per la preparazione dello standard. L'autocampionatore inietta per ogni lettura 20 microlitri.

Il programma termico prevede una prima fase di essiccamento fino a $120^{\circ} \mathrm{C}$ in 90 ", una fase di incenerimento divisa in una prima sosta a $700^{\circ} \mathrm{C}$ ed una seconda a $1400^{\circ} \mathrm{C}$ per un tempo complessivo di $55^{\prime}$, infine l'atomizzazione a $2600{ }^{\circ} \mathrm{C}$.

Complessivamente una lettura richiede un tempo di 3 minuti e 15 secondi. La calibrazione utilizza il metodo "Standard addition" con letture in "Peak area"; di ogni campione sono programmate tre repliche. Gas inerte: Argon. Il laboratorio partecipa al programma di "controllo di qualità" esterno effettuato dall'Istituto Superiore di Sanità.

\section{Metodi di calcolo}

a) Calcolo del valore medio e della deviazione standard su tutti i valori ottenuti (406 valori - 203 coppie);

b) calcolo del valore medio e della devia- 
zione standard sui valori divisi per fasce: fino a $30 \mathrm{mcg} / \mathrm{L}$, tra 30 e $60 \mathrm{mcg}$ e oltre 60 $\mathrm{mcg} / \mathrm{L}$; la divisione nelle tre fasce di valori è resa possibile dal fatto che i campioni sono stati diluiti sempre con lo stesso rapporto;

c) per verificare lo scarto tra le coppie abbiamo confrontato la media dei valori bassi (valore "A") con la media dei valori alti (valore "B") di ogni coppia; tale calcolo è stato fatto sia su tutti i valori che sugli stessi divisi nelle tre fasce: fino a $30 \mathrm{mcg} / \mathrm{L}$, tra 30 e $60 \mathrm{mcg} / \mathrm{L}$ ed oltre 60 $\mathrm{mcg} / \mathrm{L}$; abbiamo usato le seguenti formule:

$$
\begin{aligned}
\mathrm{CV} & =\frac{\mathrm{S}}{\mathrm{M}} \times 100 ; \\
\mathrm{S} & =\mathrm{M}-\mathrm{Ma} \\
\mathrm{S} & =\mathrm{Mb}-\mathrm{M}
\end{aligned}
$$

$\mathrm{CV}=$ Coefficiente di Variazione;

$\mathrm{M}=\operatorname{Media}(\mathrm{mcg} / \mathrm{L})$;

$\mathrm{Ma}=$ Media dei valori bassi delle coppie $(\mathrm{mcg} / \mathrm{L})$;

$\mathrm{Mb}=$ Media dei valori alti delle coppie $(\mathrm{mcg} / \mathrm{L})$;

$\mathrm{S}=$ Scarto tra le coppie;

d) per valutare l'affidabilità del metodo di laboratorio, come già riferito, si è proceduto alla formazione di due pool di sieri a diversa concentrazione di alluminio (pool "A" e pool "B"), e di questi un campione veniva inserito nella serie giornaliera; dopo 50 determinazioni abbiamo valutato il valore medio, la deviazione standard ed il coefficiente di variazione sia sul pool A che sul pool B.

\section{Risultati}

Le Tabelle I e II mostrano i risultati ottenuti; accanto ad ogni paziente sono segnati i valori di ogni singola coppia (il prelievo è fatto in due provette).

La Tabella III mostra l'elaborazione statistica di questi dati. Esaminando tutti i valori abbiamo ottenuto una media di $35.9 \mathrm{mcg} / \mathrm{L}$ con una deviazione standard di $32.4 \mathrm{mcg} / \mathrm{L}$ (range 5- 194 $\mathrm{mcg} / \mathrm{L}$ ); lo scarto tra le coppie è stato di $1.16 \mathrm{mcg}$ per un coefficiente di variazio-

\begin{tabular}{|c|c|c|c|c|c|c|c|c|c|c|c|}
\hline \multirow{2}{*}{$\begin{array}{c}\text { N. } \\
1\end{array}$} & \multirow{2}{*}{$\begin{array}{l}\mathrm{S} \\
\mathrm{e} \\
\mathrm{x} \\
\mathrm{F}\end{array}$} & \multirow{2}{*}{$\begin{array}{c}\text { Anz. } \\
\text { dia. } \\
\text { mesi }\end{array}$} & \multirow{2}{*}{$\begin{array}{c}\text { Età } \\
\text { anni }\end{array}$} & \multicolumn{2}{|c|}{$\begin{array}{c}\text { Al-s } \\
1^{\mathrm{a}} \\
\text { coppia }\end{array}$} & \multicolumn{2}{|c|}{$\begin{array}{c}\text { Al-s } \\
2^{\mathrm{a}} \\
\text { coppia }\end{array}$} & \multicolumn{2}{|c|}{$\begin{array}{c}\text { Al-s } \\
3^{\mathrm{a}} \\
\text { coppia }\end{array}$} & \multicolumn{2}{|c|}{$\begin{array}{c}\text { Al-s } \\
4^{\mathrm{a}} \\
\text { coppia }\end{array}$} \\
\hline & & & & 9 & 10 & 8 & 7.5 & 12 & 13 & & \\
\hline 2 & M & 6 & 49 & 10 & 12 & 8 & 9 & & & & \\
\hline 3 & $\mathrm{~F}$ & 81 & 60 & 12 & 10 & 12 & 10 & 7 & 8 & & \\
\hline 4 & $\mathrm{~F}$ & 13 & 52 & 8 & 7 & 11 & 12 & 13 & 12 & & \\
\hline 5 & M & 36 & 74 & 8 & 10 & 8 & 8 & 16 & 16 & & \\
\hline 6 & M & 22 & 66 & 9 & 10 & 7 & 7 & 16 & 18 & & \\
\hline 7 & M & 42 & 57 & 14 & 16 & 13 & 12 & 8 & 7 & & \\
\hline 8 & $\mathrm{~F}$ & 49 & 66 & 14 & 15 & 12 & 8 & 10 & 12 & & \\
\hline 9 & M & 10 & 49 & 19 & 14 & 9 & 11 & 12 & 11 & & \\
\hline 10 & M & 10 & 52 & 10 & 11 & 21 & 21 & 7 & 6 & & \\
\hline 11 & M & 17 & 65 & 11 & 9 & 14 & 12 & 18 & 18 & & \\
\hline 12 & M & 27 & 26 & 18 & 20 & 11 & 12 & 13 & 14 & & \\
\hline 13 & $\mathrm{~F}$ & 6 & 39 & 9 & 12 & 10 & 13 & 21 & 24 & & \\
\hline 14 & M & 39 & 57 & 16 & 17 & 11 & 11 & 22 & 21 & & \\
\hline 15 & M & 6 & 62 & 21 & 15 & 15 & 16 & & & & \\
\hline 16 & M & 18 & 81 & 21 & 22 & 14 & 12 & 16 & 16 & & \\
\hline 17 & M & 26 & 53 & 28 & 33 & 16 & 19 & 12 & 11 & 10 & 11 \\
\hline 18 & M & 28 & 28 & 17 & 18 & 33 & 35 & 22 & 24 & & \\
\hline 19 & M & 30 & 71 & 18 & 18 & 13 & 22 & 20 & 25 & & \\
\hline 20 & M & 18 & 56 & 20 & 22 & 13 & 15 & 24 & 25 & & \\
\hline 21 & $\mathrm{~F}$ & 32 & 65 & 32 & 33 & 16 & 21 & 11 & 7 & & \\
\hline 22 & M & 16 & 25 & 19 & 19 & 14 & 15 & 26 & 28 & & \\
\hline 23 & M & 59 & 44 & 16 & 18 & 16 & 17 & 27 & 29 & & \\
\hline 24 & M & 4 & 69 & 40 & 44 & 10 & 15 & 12 & 13 & & \\
\hline 25 & M & 36 & 29 & 27 & 28 & 19 & 20 & 22 & 20 & & \\
\hline 26 & $\mathrm{~F}$ & 84 & 48 & 23 & 24 & 16 & 22 & 28 & 29 & & \\
\hline 27 & $\mathrm{M}$ & 65 & 54 & 24 & 36 & 23 & 23 & 23 & 24 & 20 & 20 \\
\hline 28 & $\mathrm{~F}$ & 103 & 40 & 32 & 34 & 18 & 21 & 21 & 22 & & \\
\hline 29 & $\mathrm{M}$ & 92 & 58 & 18 & 22 & 26 & 37 & 22 & 24 & & \\
\hline 30 & M & 134 & 61 & 30 & 47 & 30 & 29 & 12 & 11 & 20 & 20 \\
\hline 31 & $\mathrm{~F}$ & 48 & 63 & 30 & 32 & 20 & 24 & 23 & 23 & & \\
\hline
\end{tabular}
ne di $3.2 \%$. L'esame dei valori divisi in

TAB. I

fasce ha dato i seguenti risultati: inferiori a $30 \mathrm{mcg} / \mathrm{L}$ : media $16.1 \mathrm{mcg}$, deviazione standard $8.1 \mathrm{mcg} / \mathrm{L}$, scarto tra le coppie di $1.02 \mathrm{mcg} / \mathrm{L}$ per un coefficiente di variazione di $5.3 \%$;

tra 30 e $60 \mathrm{mcg} / \mathrm{L}$ : media $42.7 \mathrm{mcg}$, deviazione standard di $12.6 \mathrm{mcg}$, scarto tra le coppie di $1.24 \mathrm{mcg}$ per un coefficiente di variazione di $2.9 \%$;

oltre $60 \mathrm{mcg} / \mathrm{L}$ : media $104.2 \mathrm{mcg}$, deviazione standard di $43.4 \mathrm{mcg}$, scarto tra le coppie di $1.69 \mathrm{mcg}$ per un coefficiente di variazione di $1.6 \%$.

La Tabella IV mostra i risultati ottenuti dal controllo interno, mediante la formazione dei due pool di sieri (A e B); dopo cinquanta determinazioni abbiamo avuto i seguenti risultati:

Pool A: media $24.6 \mathrm{mcg} / \mathrm{L}$; DS 1.0 $\mathrm{mcg} / \mathrm{L} ; \mathrm{CV} 4.0 \%$;

Pool B: media $96.5 \mathrm{mcg} / \mathrm{L}$; DS 4.0 $\mathrm{mcg} / \mathrm{L} ; \mathrm{CV} 4.1 \%$.

Nella Figura 1 è rappresentato il comportamento del Laboratorio di Chimica di Castrovillari nel controllo di qualità condotto su scala nazionale dall'Istituto Superiore di Sanità, negli anni 19921993. Le due linee delimitano l'area compresa tra $-10 \%$ e $+10 \%$ del valore Mediana risultante dall'analisi di tutti i laboratori partecipanti al controllo. I risulati dei singoli laboratori sono considerati attendibili se rientrano in questa area. Nella Figura 1 sono indicati solo i valori dati dal laboratorio di Castrovillari e come si può vedere soltanto una volta cadono fuori dall'area sopra indicata. 
TAB. II

\begin{tabular}{|c|c|c|c|c|c|c|c|c|c|}
\hline \multirow{2}{*}{$\begin{array}{l}\text { N. } \\
32\end{array}$} & \multirow[t]{2}{*}{$\begin{array}{l}\mathrm{S} \\
\mathrm{e} \\
\mathrm{x}\end{array}$} & \multirow{2}{*}{$\begin{array}{c}\begin{array}{c}\text { Anz. } \\
\text { dia. } \\
\text { mesi }\end{array} \\
67\end{array}$} & \multirow{2}{*}{$\begin{array}{c}\text { Età } \\
\text { anni } \\
64\end{array}$} & \multicolumn{2}{|c|}{$\begin{array}{c}\text { Al-s } \\
1^{\mathrm{a}} \\
\text { coppia }\end{array}$} & \multicolumn{2}{|c|}{$\begin{array}{c}\text { Al-s } \\
2^{\mathrm{a}} \\
\text { coppia }\end{array}$} & \multicolumn{2}{|c|}{$\begin{array}{c}\text { Al-s } \\
3^{\mathrm{a}} \\
\text { coppia }\end{array}$} \\
\hline & & & & 16 & 19 & 25 & 26 & 35 & 35 \\
\hline 33 & M & 79 & 68 & 31 & 31 & 23 & 22 & & \\
\hline 34 & M & 36 & 45 & 44 & 48 & 24 & 25 & 21 & 22 \\
\hline 35 & M & 64 & 46 & 25 & 30 & 42 & 40 & 13 & 13 \\
\hline 36 & M & 61 & 58 & 27 & 29 & 32 & 34 & 21 & 21 \\
\hline 37 & M & 26 & 30 & 25 & 29 & 28 & 29 & & \\
\hline 38 & $\mathrm{M}$ & 108 & 51 & 22 & 28 & 22 & 22 & 36 & 38 \\
\hline 39 & $\mathrm{~F}$ & 26 & 52 & 26 & 28 & 24 & 26 & 32 & 33 \\
\hline 40 & M & 28 & 56 & 22 & 20 & 28 & 30 & 40 & 42 \\
\hline 41 & M & 89 & 56 & 24 & 26 & 34 & 36 & 38 & 40 \\
\hline 42 & M & 51 & 62 & 31 & 33 & 44 & 46 & 28 & 29 \\
\hline 43 & $\mathrm{~F}$ & 42 & 34 & 32 & 31 & 38 & 50 & 28 & 29 \\
\hline 44 & M & 65 & 52 & 34 & 35 & 38 & 40 & 26 & 35 \\
\hline 45 & $\mathrm{~F}$ & 121 & 50 & 30 & 42 & 36 & 38 & 31 & 32 \\
\hline 46 & M & 118 & 41 & 42 & 43 & 28 & 28 & 48 & 46 \\
\hline 47 & $\mathrm{~F}$ & 116 & 72 & 40 & 42 & 37 & 38 & 36 & 37 \\
\hline 48 & $\mathrm{~F}$ & 44 & 64 & 35 & 34 & 44 & 46 & 41 & 43 \\
\hline 49 & $\mathrm{~F}$ & 87 & 57 & 58 & 61 & 39 & 44 & 29 & 30 \\
\hline 50 & M & 10 & 62 & 52 & 60 & 51 & 52 & 27 & 27 \\
\hline 51 & M & 44 & 46 & 47 & 48 & 58 & 60 & 62 & 64 \\
\hline 52 & $\mathrm{~F}$ & 55 & 63 & 60 & 64 & 40 & 41 & 66 & 69 \\
\hline 53 & $\mathrm{~F}$ & 135 & 62 & 72 & 75 & 55 & 56 & 46 & 48 \\
\hline 54 & M & 74 & 62 & 59 & 61 & 58 & 60 & & \\
\hline 55 & $\mathrm{~F}$ & 97 & 58 & 60 & 59 & 71 & 72 & & \\
\hline 56 & $\mathrm{~F}$ & 45 & 45 & 75 & 90 & 64 & 65 & 58 & 60 \\
\hline 57 & M & 37 & 45 & 90 & 92 & 54 & 55 & 67 & 70 \\
\hline 58 & M & 135 & 71 & 94 & 92 & 92 & 96 & 121 & 128 \\
\hline 59 & $\mathrm{~F}$ & 19 & 68 & 120 & 122 & 98 & 101 & 118 & 120 \\
\hline 60 & $\mathrm{~F}$ & 21 & 53 & 117 & 122 & 130 & 136 & 104 & 107 \\
\hline 61 & M & 73 & 70 & 92 & 100 & 56 & 60 & 176 & 169 \\
\hline 62 & M & 92 & 66 & 110 & 115 & 135 & 140 & 155 & 146 \\
\hline 63 & $\mathrm{~F}$ & 5 & 39 & 8 & 11 & & & & \\
\hline 64 & M & 3 & 52 & 18 & 18 & & & & \\
\hline 65 & $\mathrm{~F}$ & 3 & 43 & 13 & 16 & & & & \\
\hline 66 & M & 15 & 69 & 11 & 10 & 5 & 6 & & \\
\hline 67 & $\mathrm{~F}$ & 3 & 54 & 8 & 9 & & & & \\
\hline 68 & M & 4 & 21 & 8 & 8 & & & & \\
\hline 69 & $\mathrm{~F}$ & 50 & 73 & 19 & 22 & 21 & 22 & 23 & 24 \\
\hline
\end{tabular}

\section{Discussione}

Negli ultimi anni si è imposto all'attenzione dei nefrologi il problema della tossicità dell'alluminio nei pazienti in trattamento dialitico. Uno degli aspetti fondamentali riguarda la necessità di dosare in modo affidabile il contenuto di $\mathrm{Al}$ nel siero dei pazienti (identificazione dei pazienti con sovraccarico), nel liquido di dialisi (acqua potabile, acqua deionizzata, liquido di dialisi già preparato) e nelle varie soluzioni per uso terapeutico che possono essere fonte di intossicazione. La presenza nell'ambiente dell'alluminio rende problematico sia il prelievo che il dosaggio.

La possibilità di utilizzare apparecchiature molto valide quali gli Spettrofotometri in Assorbimento Atomico ha reso relativamente facile l'approccio al dosaggio dell'Al nei liquidi fisiologici e di interesse clinico.

Rimane da risolvere il problema della contaminazione da parte dell'ambiente.
Il nostro modello oganizzativo, nato dall'esame delle problematiche inerenti al dosaggio di questo elemento, si è mostrato molto utile in sede di prima esperienza. Dall'esame dei nostri risultati, nelle Tabelle I e II sono illustrate le "coppie" di valori di ogni paziente, si intuisce che il nostro laboratorio identifica con notevole precisione il valore dei singoli pazienti, infatti i valori delle due provette "cieche all'operatore" sono pressoché identici. Inoltre quasi tutti i pazienti (solo 5 pazienti hanno un solo controllo) sono stati sottoposti almeno 2-3 volte al dosaggio, e come si può vedere, le comprensibili differenze tra una coppia e l'altra non sono tali da far modificare il giudizio clinico, sul singolo paziente, in merito alla presenza o meno di sovraccarico dell'elemento.

La precisione del metodo di laboratorio è maggiore con i sieri a maggiore concentrazione, infatti il coefficiente di variazione si riduce passando dai valori bassi a quelli alti e ciò è dovuto al fatto che una differenza di pochi $\mathrm{mcg} / \mathrm{L}$ tra due campioni è percentualmente meno importante per i sieri a concentrazione elevata di alluminio.

Il controllo interno, attraverso la formazione dei due pool di sieri (A e B) a diversa concentrazione di alluminio ha dato buoni risultati (Tab. IV).

Il controllo esterno effettuato dall'Istituto Superiore di Sanità (Fig. 1) ha dato risultati soddisfacenti.

Concludendo, per potter ottenere dei risultati attendibili in merito al dosaggio degli elementi in tracce e nel nostro caso dell'alluminio, occorrono sì apparecchiature e materiali idonei, ma è fondamentale la cura con cui si procede al prelievo ed anche l'attenzione posta in sede di analisi.

Dalla nostra esperienza possiamo trarre alcuni dati significativi:

a) il prelievo è stato effettuato a digiuno e dopo almeno tre giorni di sospensione dell'eventuale assunzione di prodotti contenenti alluminio; da uno studio in merito condotto dal Dr. Di Iorio di Lauria $(\mathrm{PZ})$ appare più utile e corretto sospendere per almeno sette giorni (dati non pubblicati), e pertanto è possibile che le differenze osservate nello stesso paziente, in controlli diversi, possano dipendere da questo aspetto;

b) il prelievo è stato fatto raccogliendo il sangue dopo il passaggio nell'ago di al- 
TAB. III

\begin{tabular}{ccccccc}
\hline $\begin{array}{c}\text { Fasce di } \\
\text { valori }\end{array}$ & $\begin{array}{c}\text { Osserv. } \\
\text { coppie }\end{array}$ & $\begin{array}{c}\mathrm{M} \pm \mathrm{DS} \\
\mathrm{mcg} / \mathrm{L}\end{array}$ & $\begin{array}{c}\mathrm{Ma} \\
\mathrm{mcg} / \mathrm{L}\end{array}$ & $\begin{array}{c}\mathrm{Mb} \\
\mathrm{mcg} / \mathrm{L}\end{array}$ & $\begin{array}{c}\mathrm{S} \\
\mathrm{mcg} / \mathrm{L}\end{array}$ & $\mathrm{CV}$ \\
\hline $\begin{array}{c}5-194 \\
\mathrm{mcg} / \mathrm{L}\end{array}$ & 203 & $35.9 \pm 32.4$ & 34.8 & 37.1 & 1.16 & $3.2 \%$ \\
$\begin{array}{c}<30 \\
\mathrm{mcg} / \mathrm{L}\end{array}$ & 122 & $16.1 \pm 8.1$ & 15.0 & 17.1 & 1.02 & $5.3 \%$ \\
$31-60$ & 57 & $41.77 \pm 12.6$ & 41.5 & 44.0 & 1.24 & $2.9 \%$ \\
$\mathrm{mcg} / \mathrm{L}$ & 24 & $104.2 \pm 43.4$ & 102.6 & 105.9 & 1.69 & $1.7 \%$ \\
$\begin{array}{c}>60 \\
\mathrm{mcg} / \mathrm{L}\end{array}$ & & & & & & \\
\hline
\end{tabular}

$\mathrm{M}=$ Media di tutti i valori $(\mathrm{A}+\mathrm{B})$ delle coppie; $\mathrm{Ma}=$ Media dei valori bassi (valore A) di ogni singola coppia; $\mathrm{Mb}=$ Media dei valori alti (valore $\mathbf{B}$ ) di ogni singola coppia; $\mathrm{S}=$ Scarto tra le coppie; $\mathrm{CV}=$ Coefficiente di Variazione.

TAB. IV - CONTROLLO INTERNO

\begin{tabular}{ccccc}
\hline $\begin{array}{c}\text { Fasce } \\
\mathrm{di} \\
\text { valori }\end{array}$ & $\begin{array}{c}\text { Numero } \\
\mathrm{di} \\
\text { determin. }\end{array}$ & $\begin{array}{c}\text { Media } \\
\mathrm{mcg} / \mathrm{L}\end{array}$ & $\begin{array}{c}\text { Deviaz. } \\
\text { standard } \\
\text { (DS) }\end{array}$ & $\begin{array}{c}\text { Coefficiente } \\
\text { di variazione } \\
(\mathrm{CV})\end{array}$ \\
\hline Pool A & 50 & 24.6 & 1.0 & $4.0 \%$ \\
Pool B & 50 & 96.5 & 4.0 & $4.1 \%$ \\
\hline
\end{tabular}

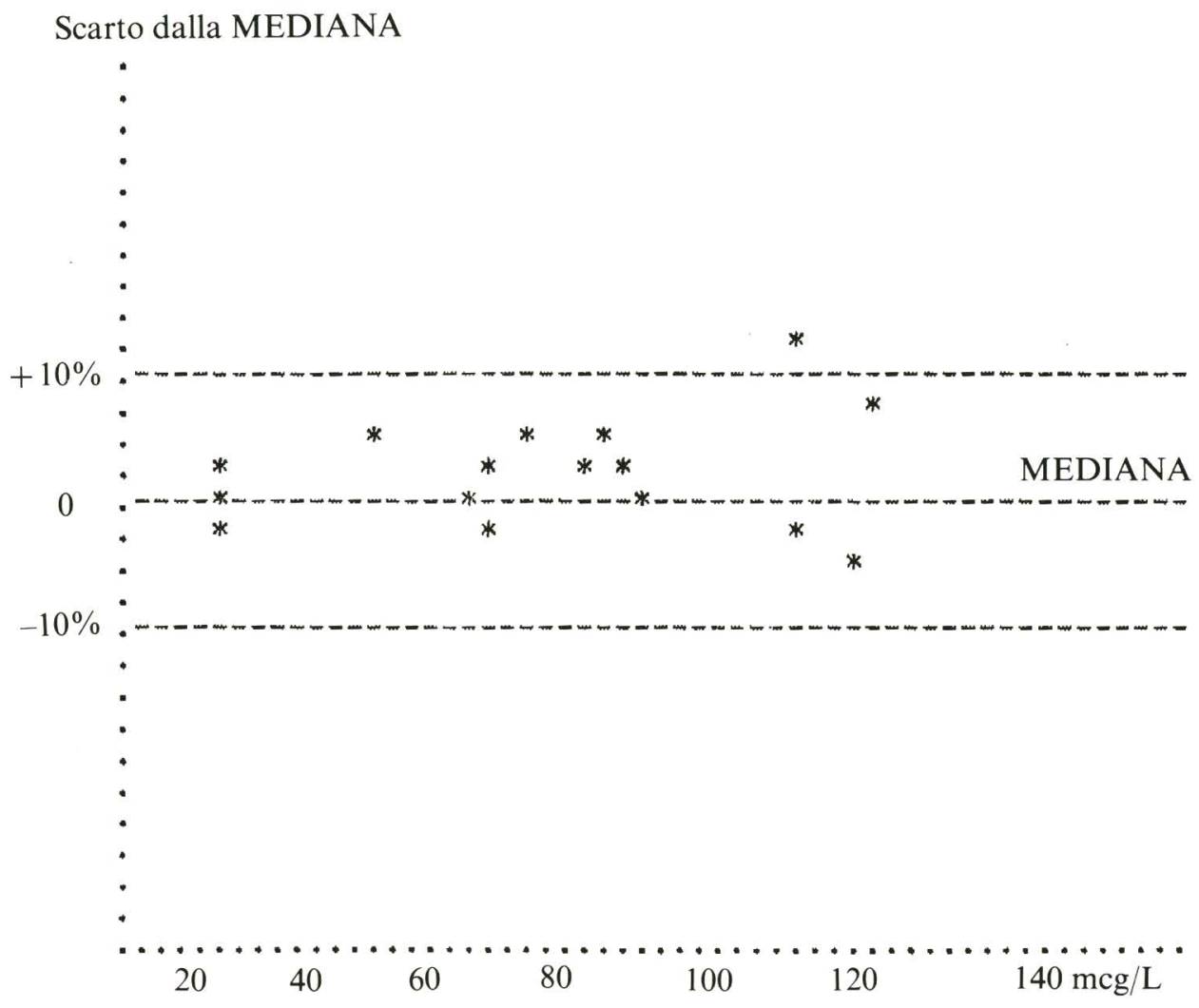

Fig. 1 - Risultati del laboratorio di Castrovillari relativi al controllo di qualità esterno condotto dall'Istituto Superiore di Sanità nel periodo 1992-1993. meno $25-30 \mathrm{ml}$; e tale sistema si è mostrato idoneo;

c) lasciare riposare le provette per almeno tre ore prima di procedere alla sieratura, rende più pulito il siero e permette di lavorare con più precisione;

d) il metodo delle "coppie" di provette "cieche" all'operatore si è mostrato molto utile per capire se i dati ottenuti fossero attendibili o meno, infatti sin dall'inizio ci si è resi conto che il nostro modello organizzativo era valido.

Noi proponiamo questo metodo e lo consigliamo ai centri dialisi ed ai laboratori che affrontano per la prima volta il dosaggio dell'alluminio.

Pur mettendo in atto i sistemi di controllo interno rimane necessario e fondamentale il controllo di qualità esterno, infatti vista l'importanza dei dati, non ci si può esimere dalla continua verifica sulla qualità dei propri risultati.

\section{BIBLIOGRAFIA}

Coen G, Mazzaferro S, Costantini $\mathrm{S}$ et al. Bone aluminium content in predialysis chronic renal failure and its relation with secondary hyperparathyroidism and $1-25(\mathrm{OH})_{2} \mathrm{D} 3$ treatment. Miner Electrolyte Metab 1989; 15 : 295-302.

Brancaccio D, GIlli P, Andriani $\mathrm{M}$ et al. L'intossicazione da alluminio nel soggetto uremico in emodialisi: ruolo del DFO. Giorn It Nefrol 1987; 2: 89-94.

Confessore A, Scarpino L, Bruno A et al. Dosaggio dell'alluminio nei pazienti dializzati: prima esperienza in un centro calabrese. Giornale di Tecniche Nefrologiche e Dialitiche 1992; 3: 2933.

4 Minoia C, Tampini G, Salvadeo $\mathrm{A}$ et al. Determinazione dell'alluminio nel siero: fattori extra analitici. Giornale Italiano Medicina del Lavoro 1984; 6: 23949. 